\title{
Exotics and all that
}

\author{
T. Barnes ${ }^{1,2}$ \\ ${ }^{1}$ Physics Division, Oak Ridge National Laboratory, Oak Ridge, TN 37831 \\ ${ }^{2}$ Department of Physics and Astronomy, University of Tennessee, Knoxville, TN 37996
}

\begin{abstract}
This invited contribution summarizes some of the more important aspects of exotics. We review theoretical expectations for exotic and nonexotic hybrid mesons, and briefly discuss the leading experimental candidate for an exotic, the $\pi_{1}(1600)$.
\end{abstract}

\section{INTRODUCTION}

An "exotic meson" is a meson resonance with $\mathrm{J}^{P C}$ or flavor quantum numbers that are forbidden to $|q \bar{q}\rangle$.

The experimental candidates of current interest are "spin-parity exotics", which have $\mathrm{J}^{P C}$ forbidden to $q \bar{q}$ mesons. In principle one might also find flavor exotics in a multiquark sector, for example in $\mathrm{I}=2$, but no widely accepted experimental candidates are known at present [1].

Every physical meson is actually a linear superposition of all allowed basis states, spanning (unless strictly forbidden) $|q \bar{q}\rangle,|q \bar{q} g\rangle,\left|q^{2} \bar{q}^{2}\right\rangle,|g g\rangle$ and so forth, with amplitudes that are determined by QCD interactions. Our working classification of resonances as "quarkonia", "hybrids", "glueballs" and so forth is a convenience that implicitly assumes that one type of basis state dominates the state expansion of each resonance. Of course this may not be the case in general, and the amount of "configuration mixing" is an important and little-studied topic in hadron physics 2]. Exotics are the special cases in which the $|q \bar{q}\rangle$ component must be zero, due to the quantum numbers of the resonance.

\section{HYBRIDS}

A hybrid meson is a resonance whose dominant valence component is $\mid q \bar{q}+$ excited glue $\rangle$. At present this is a somewhat imprecise and model-dependent definition, as there are several dissimilar models of the nature of gluonic excitations in the low energy, nonperturbative regime. The best known of these descriptions of excited glue are the bag model, constituent-gluon models, and the flux-tube model. Fortunately these very different intuitive pictures of excited glue lead to rather similar predictions for the masses and quantum numbers of lowlying hybrids.

One general conclusion is that unlike $q \bar{q}$, all $\mathrm{J}^{P C}$ quantum numbers are spanned by hybrids. This can be seen either by considering explicit models or through an enumeration of all $\left(\bar{\psi} \Gamma \lambda^{a} / 2 \psi\right) \otimes F^{a}$ interpolating fields. The list of allowed hybrid quantum numbers thus includes $\mathrm{J}^{P C}$ combinations forbidden to $q \bar{q}$ states, which are called "spin-parity exotics";

$$
\left.\mathrm{J}^{P C}\right|_{\text {exotic }}=0^{--}, 0^{+-}, 1^{-+}, 2^{+-}, 3^{-+}, \ldots .
$$

These quantum numbers are extremely attractive experimentally, since such resonances cannot be ordinary $q \bar{q}$ quark model states.

\section{A. Models of hybrids}

\section{Introduction}

Much of the work on hybrids has made use of specific models of "excited glue", especially the bag model, constituent-gluon, and flux-tube model. $\mathrm{J}^{P C}$-exotic hybrids may also be studied without specializing to a model through QCD sum rules and LGT. We will summarize some of the predictions of these models and techniques, specifically the mass spectrum, quantum numbers and decay properties.

\section{Bag model}

The bag model assumes a spherical hadron, with quarks and gluons populating cavity modes that are confined by boundary conditions on the colored quark (Dirac) and gluon (Maxwell) fields. The "zeroth-order" bag model basis states are color-singlet products of quarks, antiquarks and gluons occupying cavity modes,

$$
|q \bar{q}\rangle, \quad|q \bar{q} g\rangle, \quad|g g\rangle, \quad\left|q^{2} \bar{q}^{2}\right\rangle, \ldots .
$$

The quark-gluon and gluon self-interactions of QCD mix these basis states, so the physical levels are linear superpositions.

Combining the lowest-lying $q, \bar{q}$ and $\left(\mathrm{J}^{P}=1^{+}\right) g$ modes, one finds the lowest bag-model hybrid multiplet

$$
\left.\mathrm{J}^{P C}\right|_{\text {bag model }}=\left(0^{-}, 1^{-}\right) \otimes 1^{+}=1^{--} ; 0^{-+}, 1^{-+}, 2^{-+} .
$$

The $1^{-+}$combination is a $\mathrm{J}^{P C}$-exotic. Without the incorporation of pQCD quark-gluon and gluon-gluon interactions these levels would be degenerate. Detailed calculations of configuration mixing between these quark + gluon basis states by pQCD interactions finds the level ordering $0^{-+}<1^{-+}<1^{--}<2^{-+}$, with a total multiplet splitting of $c a$. $500 \mathrm{MeV}$, and an exotic mass of $M\left(1^{-+}\right) \approx 1.5 \mathrm{GeV}[3]$. As each of these $\mathrm{J}^{P C}$ levels is 
a flavor nonet in the $u, d, s$ system, the bag model predicts many exotic and nonexotic hybrids at relatively low masses that might be experimentally accessible.

\section{Flux-tube model}

In LGT simulations a roughly cylindrical region of modified glue fields can be observed between widely separated static color sources [4]. This "flux tube" is the origin of the confining linear potential between $q$ and $\bar{q}$ in a color-singlet $q \bar{q}$ meson. The flux-tube model [5] is an approximate description of this state of glue, which is treated as a string of point masses, "beads", connected by a linear potential. This system supports locally transverse excitations that are treated quantum mechanically by solving the bead Schrödinger equation. The orbital angular momentum carried by this model flux tube is combined with the $q \bar{q}$ spin and orbital angular momentum to form states of definite overall $\mathrm{J}^{P C}$. The lowest flux-tube hybrid multiplet spans $8 \mathrm{~J}^{P C}$ levels, with

$$
\left.\mathrm{J}^{P C}\right|_{\text {flux-tube hybrids }}=1^{ \pm \pm} ; 0^{ \pm \mp}, 1^{ \pm \mp}, 2^{ \pm \mp} .
$$

The first 2 levels have $S_{q \bar{q}}=0$ and the remainder have $S_{q \bar{q}}=1$. Note that this is a doubling of the bag model states listed earlier, with the second set having the opposite $(C, P)$. These states are all degenerate in the simplest version of the flux-tube model.

This model typically finds rather higher hybrid masses than the bag model. Isgur, Kokoski and Paton [ $[$ ] used small-oscillation and adiabatic approximations and found the lightest hybrid multiplet at 1.9(1) GeV; a subsequent Hamiltonian Monte Carlo study [7] found a very similar mass of $1.8-1.9 \mathrm{GeV}$ for this multiplet.

Since each of these $8 \mathrm{~J}^{P C}$ levels spans a flavor nonet, the flux-tube model predicts a very rich spectrum, with 72 meson resonances expected in the vicinity of $1.9 \mathrm{GeV}$, in addition to the conventional $q \bar{q}$ quark-model states!

\section{LGT and QCD Sum Rules}

LGT and QCD sum rules estimate masses by evaluating the correlation functions $\left\langle 0\left|\mathcal{O}(\vec{x}, \tau) \mathcal{O}^{\dagger}(0,0)\right| 0\right\rangle$, where $\mathcal{O}^{\dagger}$ is an operator that excites the state of interest from the vacuum. This approach uses the fact that these correlation functions summed over $\vec{x}$ at large $\tau$ approach an exponential in the mass of the lightest state excited by the operator $\mathcal{O}^{\dagger}$.

Both of these methods have systematic errors. QCD sum rules relate these correlation functions to pQCD contributions and VEVs of other operators that are inferred from experiment, and different choices for these VEVs and uncertainties in higher-mass contributions (and algebra errors) have led to a wide scatter of results in the literature. QCD sum rule mass estimates for the light $1^{-+}$exotic range from $\approx 1 \mathrm{GeV}$ to $2.1 \mathrm{GeV}$, with the higher masses preferred by the more recent references 8]. A few other exotics have been studied using QCD sum rules; the $0^{--}$for example has been found to have a rather high mass of $c a .3 \mathrm{GeV}$.

LGT results for $0^{++}$glueballs and $1^{-+}$exotic hybrids were recently reviewed by McNeile [9], and a detailed review of the approach has been published by Bali [4]. Most LGT studies to date have used the "quenched approximation", which neglects the effects of coupling to decay channels. Unfortunately these effects may include important mass shifts. Exotic hybrid masses have been studied by several groups, recently including the MILC collaboration [10] (light $1^{-+}$and $0^{+-}$exotics), UKQCD [11] $\left(0^{+-}, 1^{-+}\right.$and $2^{+-}$; these are the three exotics predicted to be lightest, and degenerate, in the zeroth-order flux-tube model), and Luo and Mei [12] (light and $c \bar{c}$ $\left.1^{-+}\right)$.

Recent LGT results are approximately consistent with the flux-tube model; signals in all three low-lying fluxtube exotic channels are observed, with the mass of the $1^{-+}$(the best determined) being about $2.0 \mathrm{GeV}$. The $0^{+-}$ and $2^{+-}$may lie somewhat higher, but this is unclear with present statistics.

The application of LGT to nonrelativistic heavy quark systems has been of much recent interest. Considerably reduced statistical errors follow from the use of an "NRQCD" action derived from a heavy quark expansion. This approach has been applied to $1^{-+}$heavy-quark exotic hybrids; the $1^{-+} b \bar{b}$ hybrid is found to lie near 11.0 $\mathrm{GeV}$, and the $1^{-+}$charmonium hybrid is predicted to lie just below $4.4 \mathrm{GeV}$. (See Ref. 12 for a summary.) These LGT results strongly motivate a high-statistics scan of $R$ near these masses, since models of hybrids anticipate that the multiplet containing the $1^{-+}$will also possess a $1^{--}$hybrid nearby in mass.

\section{B. Hybrid baryons}

One may also form hybrid baryon from $q q q$ and excited glue. Bag model calculations [13] predict a lowest multiplet of $u, d$ hybrid baryons with $\left(\mathrm{J}^{P}\right.$, flavor) $\left(1 / 2^{+} \mathrm{N}\right)^{2},\left(3 / 2^{+} \mathrm{N}\right)^{2},\left(5 / 2^{+} \mathrm{N}\right),\left(1 / 2^{+} \Delta\right),\left(3 / 2^{+} \Delta\right)$. Calculations of configuration mixing through quark-gluon and gluon-gluon interactions predict a rather large overall multiplet splitting of $c a$. $500 \mathrm{MeV}$. The resulting lowest-lying hybrid baryon is found to be a $\left(1 / 2^{+} \mathrm{N}\right)$ level, with a mass near $1.5 \mathrm{GeV}$. Recent flux-tube model calculations of hybrid baryons [14] find a rather similar spectrum of low-lying states, starting with degenerate $\left(1 / 2^{+} \mathrm{N}\right)^{2}$ and $\left(3 / 2^{+} \mathrm{N}\right)^{2}$ states at $1870(100) \mathrm{MeV}$, followed by $\left(1 / 2^{+} \Delta\right),\left(3 / 2^{+} \Delta\right)$ and $\left(5 / 2^{+} \Delta\right)$. Unfortunately there are no baryon $\mathrm{J}^{P}$-exotics, so searches for these levels must establish an overpopulation of experimental baryons relative to the theoretical $q q q$ quark model spectrum. 


\section{HYBRID DECAYS}

There is general theoretical agreement that hybrid resonances exist, and that the lightest $u, d$ hybrid meson multiplet includes a $1^{-+}$resonance with a mass in the 1.5-2 GeV region. Theoretical predictions of the strong decay widths of these states are of great interest, since many otherwise experimentally attractive decay channels may have weak couplings to hybrids, or may couple dominantly to hybrids that are so broad as to be difficult to identify, a problem familiar from the $f_{0}$ sector.

Several strong decay models been applied to hybrids. The best known is the flux-tube decay model, which was applied to exotic hybrids by Isgur, Kokoski and Paton 6] and subsequently to nonexotic hybrids by Close and Page [15]. This model assumes that decays take place by ${ }^{3} \mathrm{P}_{0} q \bar{q}$ pair production along the length of the flux tube. For the unexcited flux tubes of conventional mesons the predictions are quite similar to the rather successful ${ }^{3} \mathrm{P}_{0}$ model; for hybrids this decay model leads to predictions of very characteristic strong decay amplitudes.

In the flux-tube decay model the orbital angular momentum of the hybrid's excited flux tube gives the $q \bar{q}$ source produced in the decay a phase dependence around the axis of the original $q \bar{q}$, and the hadronic final states produced most strongly are those which have similar angular dependence. As a result, many of the well-studied simple final states such as $\pi \pi, \rho \pi$ and so forth are predicted to be produced quite weakly in hybrid decays, due to poor spatial overlap with this $e^{i \phi}$-dependent $q \bar{q}$ source. The favored modes are those that have a large $\mathrm{L}_{z}=1$ axial projection, such as an $\mathrm{S}+\mathrm{P}$ meson pair. This is the origin of the flux-tube $\mathrm{S}+\mathrm{P}$ selection rule, which in the $\mathrm{I}=11^{-+}$case favors the unusual modes $\pi f_{1}$ and $\pi b_{1}$ over $\eta \pi, \eta^{\prime} \pi$ and $\rho \pi$, despite their more limited phase space. Caution is appropriate here, since recent studies of the decay modes of orbitally-excited quarkonia in the ${ }^{3} \mathrm{P}_{0}$ model also find a preference for $\mathrm{S}+\mathrm{P}$ modes in many cases [16].

Hybrid strong decays have also been studied using QCD sum rules [8], a vector flux-tube model [17], and constituent-gluon models [18]. There is agreement (with some variation between models) that in most cases $\mathrm{S}+\mathrm{P}$ modes dominate hybrid strong decays.

Due to the difficulty of treating strong decays on the lattice there have been few studies of this very important subject. One recent, very interesting result is a LGT study of closed-flavor strong decays of heavy-quark hybrids, by McNeile, Michael and Pennanen [19]. This work finds that hybrid strong decays of the type $1^{-+} \rightarrow \chi S$, where $\chi$ is a $\mathrm{P}$-wave $Q \bar{Q}$ and $S$ is a light $q \bar{q}$ scalar, are much larger than expected; partial widths in the 10s of $\mathrm{MeV}$ appear likely. This is excellent news for experimental searches, since transitions such as $H_{c} \rightarrow \chi_{c} S \rightarrow$ $\gamma J / \psi(\pi \pi)_{S}, J / \psi \rightarrow \ell^{+} \ell^{-}$allow efficient background rejection. Closed-flavor strong cascades had been suggested previously as a method for searching for heavy hybrids in $e^{+} e^{-}$annihilation (see for example Ref.[20]), but it was thought that the transition rates would be much smaller. If the new LGT results are correct, this approach now appears very attractive.

\section{AN EXPERIMENTAL EXOTIC MESON: $\pi_{1}(1600)$}

At present there are just two experimental candidates for exotic mesons, the $\pi_{1}(1400)$ and the $\pi_{1}(1600)$. In view of the limited space available here, I will only discuss the well established $\pi_{1}(1600)$. The long and complicated history of the $\pi_{1}(1400)$ is summarized elsewhere [21].

Evidence for the $\mathrm{I}=1, \mathrm{~J}^{P C}=1^{-+} \pi_{1}(1600)$ has been reported in three channels, $b_{1} \pi$ (VES 22]), $\eta^{\prime} \pi$ (VES 22] and E852 at BNL [23]) and $\rho \pi$ (VES [22] and E852 [24]). Clear resonant phase motion is seen relative to the wellknown $q \bar{q}$ states $a_{2}(1320)$ and $\pi_{2}(1670)$ in the $\eta^{\prime} \pi$ and $\rho \pi$ channels respectively. The mass and width reported by VES and E852 are consistent,

$$
M_{\pi_{1}}= \begin{cases}1.61(2) \mathrm{GeV} & \text { VES, all modes } \\ 1.597 \pm 0.010_{-0.010}^{+0.045} \mathrm{GeV} & \mathrm{E} 852, \eta^{\prime} \pi \\ 1.593 \pm 0.008_{-0.047}^{+0.029} \mathrm{GeV} & \mathrm{E} 852, \rho \pi\end{cases}
$$

$\Gamma_{\pi_{1}}= \begin{cases}0.29(3) \mathrm{GeV} & \text { VES, all modes } \\ 0.340 \pm 0.040 \pm 0.050 \mathrm{GeV} & \mathrm{E} 852, \eta^{\prime} \pi \\ 0.168 \pm 0.020_{-0.012}^{+0.150} \mathrm{GeV} & \mathrm{E} 852, \rho \pi\end{cases}$

The $\pi_{1}(1600)$ signal is especially clear in $\eta^{\prime} \pi$, in part because $q \bar{q}$ states such as the $a_{2}(1320)$ have small branching fractions to this channel. (See Fig.2 of Ref. [23].)

The relative $\pi_{1}(1600)$ branching fractions reported by VES for the final states $b_{1} \pi, \eta^{\prime} \pi$ and $\rho \pi$ are

$$
\Gamma\left(\pi_{1}(1600) \rightarrow f\right)= \begin{cases}\equiv 1 & b_{1} \pi \\ 1.0 \pm 0.3 & \eta^{\prime} \pi \\ 1.6 \pm 0.4 & \rho \pi\end{cases}
$$

Although the $\pi_{1}(1600)$ is a well-established exotic resonance, there are problems with identifying it with a hybrid. One difficulty is the $\approx 300-400 \mathrm{MeV}$ difference between flux-tube and LGT estimates of $M \approx 1.9-2.0 \mathrm{GeV}$ and the $\pi_{1}(1600)$ mass. This discrepancy might of course be a result of the quenched approximation used in LGT.

A second problem with teh hybrid assignment is that the reported relative branching fractions are inconsistent with the predictions of the flux-tube model that $\mathrm{S}+\mathrm{P}$ modes should be dominant. For this state the flux-tube model predicts that $b_{1} \pi$ should be dominant, with $\rho \pi$ weak and $\eta \pi$ and $\eta^{\prime} \pi$ very small [6, 15]. Some $\rho \pi$ coupling is expected in the flux-tube model due to different $\rho$ and $\pi$ spatial wavefunctions [15], but this is expected to be a much smaller effect in the $\eta \pi$ and $\eta^{\prime} \pi$ modes. Indeed, there is a generalized G-parity argument that these $\mathrm{S}+\mathrm{S}$ 
partial widths would be zero except for differences in the final spatial wavefunctions 25]. Either these three modes are not all due to a hybrid, or our understanding of hybrid decays is inaccurate.

Future experimental studies of the $\pi_{1}(1600)$ in all its allowed strong decay modes will be especially interesting as tests of theoretical models of exotic meson decays. This state is of special relevance for the GlueX photoproduction facility planned at Jefferson Laboratory [26, 27], since the $\pi_{1}(1600)$ and other resonances with significant $\rho \pi$ couplings should be produced copiously in one-pion- exchange photoproduction processes.

\section{ACKNOWLEDGMENTS}

This work was supported in part by the United States Department of Energy under contract DE-AC0596OR22464 managed by UT Battelle at Oak Ridge National Laboratory, and by the University of Tennessee.
[1] For a recent review of light meson spectroscopy see S.Godfrey and J.Napolitano, Rev. Mod. Phys. 71, 1411 (1999), hep-ph/9811410

[2] T. Burch, K. Orginos and D. Toussaint, Nucl. Phys. Proc. Suppl. 106, 382 (2002), hep-lat/0110001

[3] T.Barnes, PhD thesis, Caltech (1977); Nucl. Phys. B158, 171 (1979); T.Barnes and F.E.Close, Phys. Lett. 116B, 365 (1982); M.Chanowitz and S.Sharpe, Nucl. Phys. B222, 211 (1983), err. B228, 588 (1983); T.Barnes, F.E.Close and F.deViron, Nucl. Phys. B224, 241 (1983).

[4] G.S.Bali, K.Schilling and C.Schlichter, Phys. Rev. D 51, 5165 (1995), hep-lat/9409005 For a review of LGT applications to hadron spectroscopy see G.S.Bali, Phys. Rept. 343, 1 (2001), hep-ph/0001312

[5] N.Isgur and J.Paton, Phys. Rev. D31, 2910 (1985).

[6] N.Isgur, R.Kokoski and J.Paton, Phys. Rev. Lett. 54, 869 (1985).

[7] T.Barnes, F.E.Close and E.S.Swanson, Phys. Rev. D52, 5242 (1995).

[8] I.I.Balitsky, D.Dyakanov and A.V.Yung, Phys. Lett. B112, $71(1982)$ quoted a $1^{-+}$mass of $\approx 1 \mathrm{GeV}$. A subsequent paper, Z. Phys. C33, 265 (1986), estimated a rather higher mass. Other QCD sum rule results for the mass of the $1^{-+}$exotic are J. Govaerts, F.deViron, D.Gusbin and J.Weyers, Nucl. Phys. B248, 1 (1984), 1.3 GeV; J.I Latorre, S.Narison, P.Pascual and R.Tarrach, Phys. Lett. B147, 169 (1984), 1.7(1) GeV; J.I.Latorre, P.Pascual and S.Narison, Z. Phys. C34, 347 (1987), 2.1 GeV. Most recently, K.Chetyrkin and S.Narison, Phys. Lett. B485, 145 (2000), hep-ph/0003151 v2 quote $\approx 1.6-1.7 \mathrm{GeV}$, with the radial hybrid only about $0.2 \mathrm{GeV}$ higher in mass. Chetyrkin and Narison also consider decay couplings; they find that the $\pi_{1}(1600-1700)$ exotic has $\Gamma_{\pi \rho} \approx 300 \mathrm{MeV}$, but for $\Gamma_{\pi \eta^{\prime}}$ only $\approx 3 \mathrm{MeV}$. This is inconsistent with the reported decays of both experimental $\pi_{1}$ exotic candidates.

[9] C.McNeile, hep-lat/0207001

[10] C.Bernard et al. (MILC Collaboration), Phys. Rev. D56, 7039 (1997).

[11] P.Lacock et al. (UKQCD Collaboration), Phys. Lett.
B401, 308 (1997).

[12] X.-Q. Luo and Z.-H. Mei, hep-lat/0209049

[13] T.Barnes and F.E.Close, Phys. Lett. 123B, 89 (1983); Phys. Lett. 128B, 277 (1983); C.E.Carlson, T.H.Hansson and C.Peterson, Phys. Rev. D27, 1556 (1983); E.Golowich, E.Haqq and G.Karl, Phys. Rev. D28, 160 (1983); err. D33, 859 (1986).

[14] S.Capstick and P.R.Page, Phys. Rev. D60, 111501 (1999), nucl-th/9904041

[15] F.E.Close and P.R.Page, Nucl. Phys. B443, 233 (1995).

[16] For $(u, d)$ meson decays see T.Barnes, F.E.Close, P.R.Page and E.S.Swanson, Phys. Rev. D55, 4157 (1996), hep-ph/9609339 For decays of strange mesons see T.Barnes, N.Black and P.R.Page, nucl-th/0208072

[17] P.R.Page, E.S.Swanson and A.P.Szczepaniak, Phys. Rev. D59, 034016 (1999).

[18] M.Tanimoto, Phys. Lett. B116, 198 (1982); A.LeYaouanc et al., Z. Phys. C28, 309 (1985); F.Iddir et al., Phys. Lett. B207, 325 (1988); B433, 125 (1998), hep-ph/9803470 Yu.S.Kalashnikova, Z. Phys. C62, 323 (1994).

[19] C.McNeile, Phys. Rev. D65, 094505 (2002), hep-lat/0201006

[20] T.Barnes, Charmonium Physics at a Tau-Charm Factory, hep-ph/9308368

[21] T.Barnes, Acta Phys. Polon. B31, 2545 (2000), hep-ph/0007296

[22] V.Dorofeev (VES Collaboration), in Proceedings of WHS99, hep-ex/9905002

[23] E.I.Ivanov et al. (E852 Collaboration), Phys. Rev. Lett. 86, 3977 (2001).

[24] G.S.Adams et al. (E852 Collaboration), Phys. Rev. Lett. 81, 5760 (1998); S.U.Chung et al., Phys. Rev. D65, 072001 (2002).

[25] P.R.Page, Phys. Lett. B402, 103 (1997).

[26] A.Afanasev and P.R.Page, Phys. Rev. D57, 6771 (1998).

[27] A. R. Dzierba, "QCD Confinement and the Hall D Project at Jefferson Lab", in Proc. of $e+e$ - Physics at Intermediate Energies, (SLAC, Stanford, California, 30 Apr - 2 May 2001), hep-ex/0106010 